\title{
Effet De Quatre Traitements De Substrat Sur L'apparition Des Maladies De La Tomate [Solanum Lycopersicum L. (Solanaceae)] En Culture Hors Sol, Côte d'Ivoire
}

\author{
Koffi Ahébé Marie Hélène \\ Yah N'guéttia Marie \\ Koffi N'dodo Bony Clovis \\ Amon Michel Wilfried
}

Université Jean Lorougnon Guédé, UFR Agroforesterie, Laboratoire d'Amélioration de la Production Agricole, Côte d'Ivoire

\section{Atta Taky Hortense Diallo}

Université Nangui Abrogoua, UFR Science de la Nature, Laboratoire d'Amélioration de la Production Végétale, Côte d'Ivoire

Doi:10.19044/esj.2021.v17n3p63

Submitted: 31 October 2020

Accepted: 25 December 2020

Published: 31 January 2021
Copyright 2021 Author(s)

Under Creative Commons BY-NC-ND

4.0 OPEN ACCESS

Cite As:

Koffi Ahébé M.H., Yah N’G. M., Koffi N’dodo B.C., Amon M.W. \& Atta Taky H.D. (2021). Effet De Quatre Traitements De Substrat Sur L'apparition Des Maladies De La Tomate [Solanum lycopersicum L. (Solanaceae)] En Culture Hors Sol, Côte d'Ivoire. European Scientific Journal, ESJ, 17(3), 63.

https://doi.org/10.19044/esj.2021.v17n3p63

\section{Résumé}

La Tomate est un légume-fruit riche en éléments nutritifs et très prisée en Côte d'Ivoire. Malgré ses nombreux bienfaits, sa production reste encore faible dans notre pays. Cette faible production est entre autre due à la forte pression parasitaire, à l'influence des facteurs abiotiques et aux pratiques agricoles rudimentaires. Dans nos travaux, l'objectif recherché était d'améliorer la production de la tomate. Pour atteindre cet objectif, une expérience portant sur la culture de la tomate en hors sol a été effectuée. A cet effet, quatre traitements de la sciure de bois ont été testés sur une culture de tomate (variété F1 cobra 26) dans le domaine expérimentale champ école de l’Université Jean Lorougnon Guédé de Daloa. Il s’agit de la sciure de bois simple constituant le témoin, de la sciure de bois mélangée avec les pesticides CALLOMIL super $66 \mathrm{WP}$, MANCOZAN $80 \mathrm{WP}$, VYTAL 3G, PYRICAL $5 G$, de la sciure de bois chauffée et de la sciure de bois chauffée plus ajout de 
pesticides. Deux semaines après repiquage $84 \mathrm{ml}$ de NPK 15-9-20 par sachets ont été utilisés pour l'arrosage des plants de chaque traitement. Les performances de ces traitements ont été appréciées à travers l'incidence de la maladie, le taux de mortalité des plants et le taux de fruit présentant des symptômes. L'incidence de la maladie est faible (5,88 \%) dans le sous bloc sciure de bois chauffé additionnée de pesticides comparativement aux autres traitements. La mortalité la plus élevée est celle du témoin (56,52 \%) suivie de la sciure de bois chauffé (34,78\%), ensuite celle traité avec des pesticides $(13,05 \%)$ et enfin le taux le plus bas (4,34 \%) avec la sciure de bois chauffée additionnée de pesticides. Le témoin a présenté le taux de fruit infecté le plus élevé (72 \%), suivie de la sciure chauffée avec un taux de symptômes de (47,61 $\%)$. La sciure traitée avec des pesticides et substrat chauffé plus pesticides ont eu de faible taux de fruit présentant des symptômes de 4,99 et 1,96 \% respectivement. La sciure de bois chauffé plus ajout des pesticides est la meilleure des quatre traitements pour la protection des plants contre les agents pathogènes.

Mots-clés: Tomate, Sciure De Bois, Maladie, Pesticides, Incidence

\title{
Effect Of Four Substrate Treatments On Tomato Diseases Occurrence [Solanum Lycopersicum L. (Solanaceae)] Off- Ground Culture, Côte d'Ivoire
}

\author{
Koffi Ahébé Marie Hélène \\ Yah N'guéttia Marie \\ Koffi N'dodo Bony Clovis \\ Amon Michel Wilfried \\ Université Jean Lorougnon Guédé, UFR Agroforesterie, Laboratoire \\ d'Amélioration de la Production Agricole, Côte d'Ivoire \\ Atta Taky Hortense Diallo \\ Université Nangui Abrogoua, UFR Science de la Nature, Laboratoire \\ d’Amélioration de la Production Végétale, Côte d’Ivoire
}

\begin{abstract}
Tomato is a nutritious fruit vegetable that is very popular in Côte d'Ivoire. Despite its many benefits, its production is still low in our country. This low production is due to high parasite pressure, the influence of abiotic factors and rudimentary agricultural practices. In our work, the aim was to improve tomato production. To reach this aim, an experiment was carried out on the cultivation of tomatoes above ground. To this end, Four wood sawdust
\end{abstract}


treatments were tested on tomato crop (variety F1 cobra 26) in the experimental field school field of University Jean Lorougnon Guede of Daloa. These are control sawdust, sawdust mixed with pesticides CALLOMIL super 66 WP, MANCOZAN 80 WP, VYTAL 3G, PYRICAL 5G, heated sawdust and heated sawdust added pesticide. Two weeks after transplanting $84 \mathrm{ml}$ of NPK 15-9-20 per sachets were used for watering plants of each treatment. The performance of these treatments was assessed through disease incidence, mortality rate, and rate of fruit showing symptoms. Incidence of the disease is low (5.88\%) in heated sawdust added pesticides sub-block compared to other treatments. The highest mortality is that of the control (56.52\%) followed by heated sawdust (34.78\%), then that treated with pesticides (13.05\%) and finally the lowest (4.34\%) with heated sawdust with added pesticides. Control showed the highest rate of infected fruit (72\%), followed by heated sawdust with symptom rate of (47.61\%). Sawdust treated with pesticides and heated substrate plus pesticides had low fruit rates with symptoms of 4.99 and $1.96 \%$ respectively. Heated sawdust added pesticides is the best of the four treatments for plants protection against pathogens.

Keywords: Tomato, Sawdust, Disease, Pesticides, Incidence

\section{Introduction}

La tomate (Solanum lycopersicum, L., Solanacée) compte parmi les cultures légumières les plus importantes du monde (Naika et al., 2005). Elle constitue le deuxième légume, le plus consommé dans le monde après la pomme de terre (INRA, 2010). Dans le secteur vivrier, les légumes jouent un rôle prépondérant dans l'alimentation quotidienne des populations. Ainsi, la tomate est l'une des espèces légumières la plus cultivée en Afrique car elle entre dans la composition de nombreux plats traditionnels (Boa, 2016). En Côte d'Ivoire, en dépit des atouts naturels qui offrent une production vivrière brute de près de 8 millions de tonnes par an, la demande en produits légumiers est croissante (Anonyme, 2005). Ainsi, la tomate occupe une place de choix tant au niveau nutritionnel et qu'économique. La production annuelle de tomate fluctue entre 22000 et 35000 tonnes (Sangaré et al., 2009). En effet, les besoins en tomates estimés à plus de 100000 tonnes ne sont couverts qu'aux deux tiers par la production locale. De même, ces besoins étaient estimés à plus de 200000 tonnes en 2002 (N'zi et al., 2010) alors que la production nationale qui est de 52000 tonnes par an reste largement inférieure pour couvrir cette demande (Soro, 2009 ; N'zi et al., 2010).

En Côte d'Ivoire, la pratique agricole de la tomate se fait selon deux modalités : plein champ et hors sol. Le mode de culture plein champ occasionne la dégradation des ressources naturelles due à une exploitation anarchique et incontrôlée des sols. En effet, 80 \% de la population tirent leurs 
revenus dans l'agriculture par l'exploitation continue des sols. Face à ces contraintes, une solution à long terme est possible à travers l'application du système de production hors-sol (Djaha, 2015). La tomate cultivée hors sol permet de maîtriser l'alimentation hydrique et minérale des plants. Cette méthode représente actuellement une technique importante sur les exploitations permettant de mieux s'adapter aux données économiques du marché en optimisant des facteurs de production agricole tout en améliorant les rendements (Bernier, 2015). En effet parmi les principales raisons d'introduction de la culture hors sol figurent l'élimination des problèmes liés aux sols, la rationalisation de l'utilisation de l'eau, des engrais et l'amélioration qualitative et quantitative du rendement (Sedki et al., 1995).

Cependant, le développement de la culture de tomate dans nos régions tropicales est confronté à d'énormes difficultés. Au cours de son cycle végétatif, la tomate est continuellement attaquée par de nombreux ravageurs et maladies occasionnant des dégâts sur les divers organes des plantes et à diverses étapes de leur croissance et développement (Bennett et al., 2003). Aussi, la forte pression parasitaire, l'influence des facteurs abiotiques et les pratiques agricoles rudimentaires réduisent fortement la productions (Nechadi et al., 2002).

La culture hydroponique a été introduite en Côte d'Ivoire en 2008 pour faciliter l'accès des populations urbaines aux produits maraîchers de qualité, réduire les difficultés d'accès à la terre, contribuer à la création d'emplois pour les jeunes et les femmes et occuper les personnes âgées. Cependant, la diffusion de cette technologie a rencontré de nombreuses difficultés (Djaha, 2015). Les producteurs font faces à de récurrents problèmes de maladies pour un système de culture qui devrait aider à surmonter la plupart des problèmes de maladies (Boa, 2016). Face à cette situation, des recherches sont effectuées pour aider à la maîtrise des maladies de la tomate hors sol. C'est dans ce cadre que s'inscrit cette étude dont l'objectif est de contribuer à l'amélioration de la culture de la tomate hors sol. Il a consisté à l'évaluation de l'incidence de différents traitements sur l'apparition des symptômes des maladies de la tomate.

\section{Matériel et Méthodes Site d'étude}

La parcelle expérimentale est située à l'Université Jean Lorougnon Guédé de Daloa. La ville de Daloa est localisée dans la région du HautSassandra, au Centre-Ouest de la Côte d'Ivoire entre le $6^{\circ}$ et $7^{\circ}$ de latitude Nord et le $7^{\circ}$ et $8^{\circ}$ de longitude Ouest (Figure 1). Le substrat pédologique de Daloa appartient au vieux socle précambrien composé de granites, migmatites. Ces sols, lessivés et profonds de $20 \mathrm{~m}$ sont la conséquence des précipitations abondantes et à l'altération rapide des roches. Les sols de la région sont 
majoritairement ferralitiques. Ils sont généralement très profonds avec un taux élevé de matière organique.

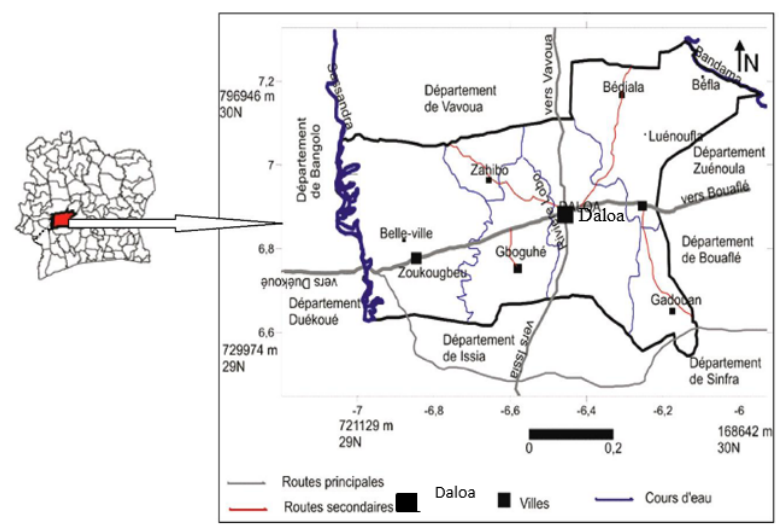

Figure 1: Zone d'étude (Source : Adjiri et al., 2019)

\section{Matériel}

\section{Matériel végétal}

Le matériel végétal a été composé de semences de tomate hybride de la variété F1 COBRA 26 et la sciure de bois décomposé âgée d'un mois (Figure 2).

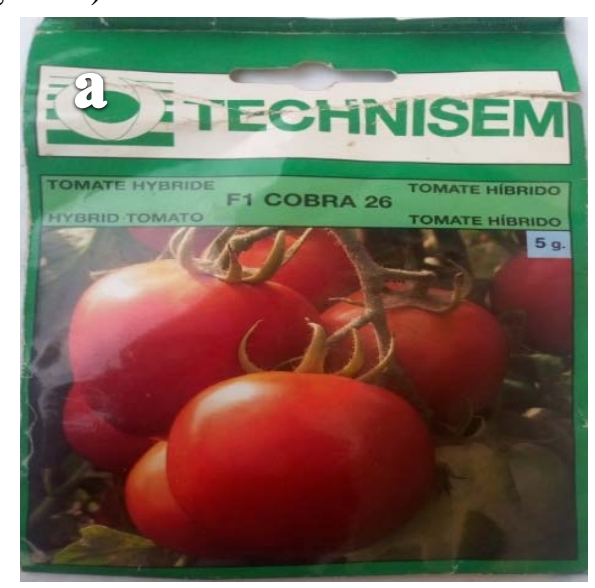

a : Semence de la variété F1 COBRA 26 ;

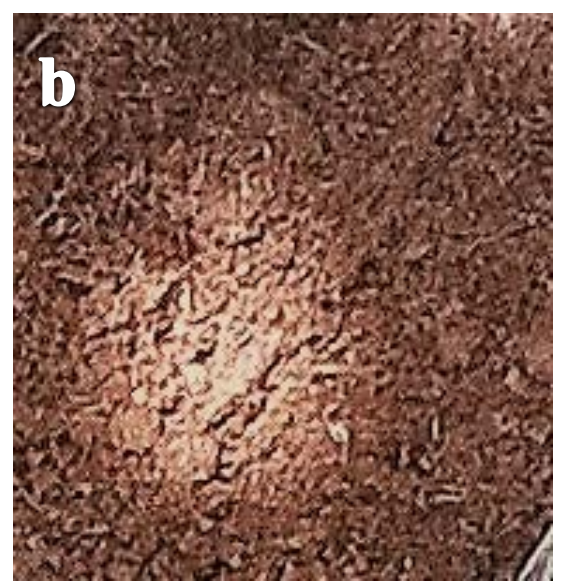

b : Sciure de bois décomposée âgée d'un mois

Figure 2. Matériel végétal utilisé

\section{Matériel chimique}

Le matériel chimique a été composé de quatre pesticides que sont la Callomil super 66 WP, le Mancozan 80 WP, le Vytal 3G, le Pyrical 5G et de l'engrais minéral NPK 15-9-20. 


\section{Méthodes}

\section{Dispositif expérimental}

Le dispositif expérimental a été constitué d'un bloc de Fischer de $9 \mathrm{~m} \mathrm{x}$ $7 \mathrm{~m}$ constitué de 4 sous blocs. Chaque sous bloc de $3 \mathrm{~m}$ x 1,5 m correspondait à un traitement de culture et a été constitué de 15 sachets de culture. L'écartement entre les sous blocs a été de $0,5 \mathrm{~m}$ et de $0,3 \mathrm{~m}$ d'un sachet à l'autre (Figure 3).

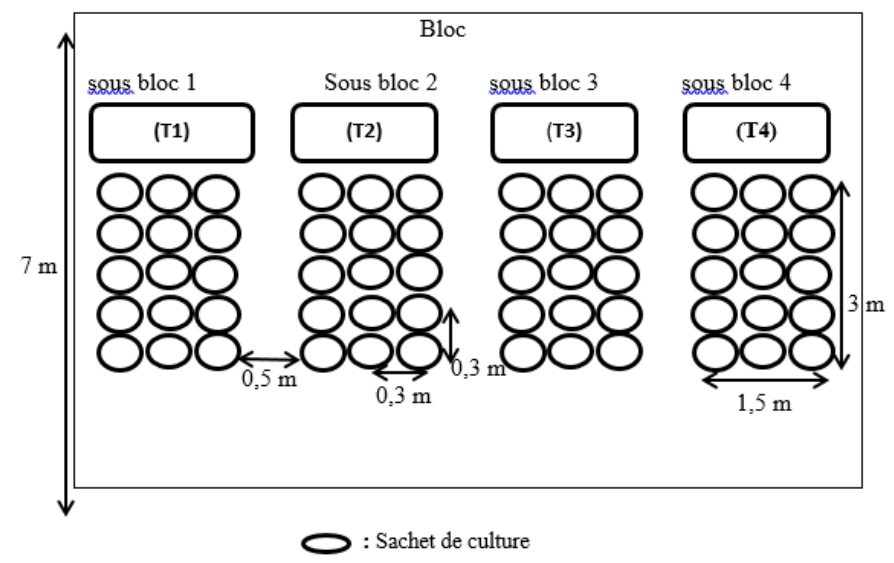

Figure 3. Dispositif expérimenta

\section{Echantillonnage et choix du matériel végétal et chimique}

Les semences de tomate hybride de la variété F1 COBRA 26 ont été achetées à SEMIVOIRE. Cette variété a été choisie d'une part, pour sa tolérance au TYLCV et au flétrissement bactérien et d'autre part, pour sa productivité

La sciure de bois décomposée a été collectée auprès d'une scierie du quartier Kennedy II de Daloa. Elle a été exposée à l’air libre sur le sol nu, sans ombrage au gré des pluies et autres intempéries pendant un mois. Son choix est basé sur sa facilité d'usage et son coût moins élevé. Le substrat décomposé a été mis dans les sachets destinés é la pépinière de coco.

Les différents pesticides et l'engrais NPK 15-9-20 ont été achetés à CALLIVOIRE sise à Daloa. En effet, la Callomil super 66 WP et le Mancozan 80 WP sont tous deux des fongicides. Cependant, la Callomil super 66 WP est systémique de contact à action préventive et curative, utiliser contre la pourriture des fruits de tomate alors que le Mancozan 80 WP est préventif de contact à large spectre d'action utilisé contre plusieurs maladies fongiques. Le Vytal 3G et le Pyrical 5g sont utilisé comme insecticide pour lutter contre les insectes du sol. Aussi, le Vytal 3G a un effet nematicide contre les nématodes à galles. Le NPK renforce la tolérance de la plante au stress et aux maladies. 


\section{Traitements des différents substrats de culture}

Quatre types de traitements ont été effectués

-Le traitement (T1) a été utilisé comme témoin. A cet effet, la sciure de bois décomposée, sans apport de pesticide ni chauffée a été mise dans 15 sachets de culture à raison de $1 \mathrm{Kg}$ de sciure par sachet.

-Le traitement (T2) a été constitué de sciure de bois chauffée pendant 45 minutes dans un seau de peinture métallique afin de réduire la population d'éventuels parasites. Après le chauffage, le contenu a été renversé sur du sachet plastique en polyéthylène pour refroidissement. Un Kg de cette sciure a été renversé dans chacune des 15 sachets de culture.

-Le traitement (T3) a été constitué de sciure de bois non chauffée additionnée de pesticides. Ainsi, un mélange constitué de 1,62 g de Callomil super 66 WP, 101,35 g de Mancozan 80 WP, 0,94 g de VytaL 3G et 0,54 g de Pyrical 5G a été ajouté à chaque sachet de culture rempli au préalable de $1 \mathrm{Kg}$ de sciure de bois.

-Le traitement (T4) associe avec le traitement thermique et chimique. La sciure de bois a été d'abord portée au feu et refroidit sur des sacs de riz. Ensuite, 1 Kg de cette sciure a été mis dans chaque sachet de culture et enfin, un mélange constitué de 1,62 g de Callomil super 66 WP, 101,35 g de Mancozan 80 WP, $0,94 \mathrm{~g}$ de Vytal $3 \mathrm{G}$ et $0,54 \mathrm{~g}$ de Pyrical 5G a été ajouté à chaque sachet de culture rempli au préalable.

\section{Mise en place et entretien de la pépinière}

Un Kilogramme de sciure de bois décomposé a d'abord été mélangé à 144 g d'engrais NPK 15-9-20 et 39 g Pyrical. Le mélange a été mis dans une plaque de 72 alvéoles à raison de deux grammes par alvéole. Ensuite, le semis a été effectué à raison de deux semences de F1 COBRA 26 par alvéole.

La plaque alvéolée a été posée sur une planche à $5 \mathrm{~cm}$ du sol et une ombrière constituée de feuilles de palme a été construite à $80 \mathrm{~cm}$ au-dessus du sol pour protéger les plants contre l'ensoleillement.

Les plants ont été régulièrement arrosés et des désherbages manuels si nécessaire ont été réalisés. Les feuilles de palme ont été retirées progressivement à 3 jours avant le repiquage pour permettre aux plants de s'adapter aux rayons lumineux avant d'être repiqués.

\section{Repiquage et suivi des plants}

Après trois semaines en pépinière, les plants apparemment sains et vigoureux ont été repiqués dans les sachets contenant les différents traitements. Deux plants ont été repiqués dans chaque sachet. Soit un total de 30 plants par traitement. Deux semaines après repiquage, 144 g d'engrais NPK 15-9-20 dilué dans 5 litres d'eau a été utilisé pour l'arrosage des plants de chaque traitement soit $83,33 \mathrm{ml}$ par sachet. Un désherbage manuel si 
nécessaire a été effectué. Les plants ont été tuteurés afin d'éviter le contact des fruits avec le sol.

\section{Descriptions des symptômes observés}

Les symptômes sur les feuilles, tiges, fruits, collets et racines ont été observés, dès leur apparition trois semaines après le repiquage. A cet effet, chaque semaine, l'aspect, la forme et la coloration des symptômes ont été décrits par observation visuelle et au touché. Le nombre de pied présentant des symptômes a également été noté à chaque observation par sous bloc et cela pour chaque traitement.

\section{Calcul des paramètres Incidence des symptômes}

L'incidence des symptômes a été évaluée pour chaque traitement de la troisième à la douzième semaine après repiquage. Pour ce faire, le nombre d'organe (pied ou feuille) malade a été compté et l'incidence de chaque symptôme a été déterminée par le rapport du nombre d'organe présentant un symptôme sur le nombre total d'organe pour chaque traitement selon la formule de Boa, 2016 :

$$
\begin{gathered}
\text { I (\%) }=\frac{\Sigma \mathbf{n}}{\mathbf{N}} \times \mathbf{1 0 0} \\
\text { I (\%): Incidence moyenne du symptôme } \\
\mathbf{n}: \text { Nombre d'organe présentant un symptôme } \\
\mathbf{N}: \text { Nombre totale d'organe par traitement avec N=30 }
\end{gathered}
$$

\section{Taux de mortalité des plants}

Après repiquage les plants ont été observés chaque semaine pendant un mois et demi, le taux de mortalité a été évalué à partir du nombre de plants morts suite aux infections par rapport aux plants infectés mais ayant survécu aux infections. Ce taux a été calculé pour chaque traitement selon la formule de Vakalounakis et Fragkiadakis (1999).

$$
\text { TM }(\%)=\frac{\Sigma \mathbf{n}}{\mathbf{N}} \times 100
$$

TM : Taux de mortalité ;

n : Nombre de plants mort

$\mathbf{N}$ : Nombre de plants présentant les symptômes par traitement avec $\mathrm{N}=30$

\section{Taux de fruit présentant des symptômes}

Lors de la production, le taux de fruits présentant des symptômes a été observé et le nombre de fruits atteint sur le nombre total de fruits pour chaque traitement a été compté. Le taux de fruit avarié a été calculé selon la formule ci-dessous (Biekre, 2013). 


$$
\operatorname{TFA}(\%)=\frac{\Sigma \mathbf{n}}{\mathbf{N}} \times 100
$$

TFA(\%): Taux de fruits présentant des symptômes n : Nombre total de fruits présentant des symptômes

$\mathbf{N}$ : Nombre totale des fruits avec $\mathrm{N}=42$

\section{Analyse statistique des données}

Une analyse de la variance à un critère de classification (ANOVA 1) a été effectuée au seuil $\alpha=0,05$. Le logiciel Statistica 7.1 a été utilisé pour tester l'effet du traitement sur l'incidence de la maladie, le taux de mortalité des plants et le taux de fruits avariés. En cas de différence significative, les moyennes ont été classées à l'aide du test de Duncan.

\section{Résultats et discussion \\ Résultats \\ Description des symptômes}

Huit types de symptômes ont été observés sur les plants des différents traitements (Tableau 1).

Le premier symptôme a été uniquement observé au niveau du traitement témoin. Il se présente sous forme de dépérissement de la tige secondaire (Figure 4a).

Trois types de symptômes ont été à la fois observés sur les plants au niveau de deux traitements à savoir le traitement témoin et le traitement sciure de bois chauffé. Ces symptômes se manifestent respectivement sous forme de nécroses sur la feuille (Figure 4b), de chancre sur le collet (Figure 4c) et de galles sur la racine (Figure $4 d$ ).

Le cinquième symptôme a été présent au niveau des plants de trois traitements que sont le témoin, la sciure de bois chauffé et le traitement sciure de bois non chauffée additionnée de pesticide. Il se présente comme une nécrose sur la tige (Figure 4e).

Les trois derniers symptômes ont été présents sur les plants au niveau de tous les quatre traitements. C'est à dire le témoin, la sciure de bois chauffé, la sciure de bois non chauffée additionnée de pesticide et la sciure de bois chauffée additionnée de pesticide. Ces symptômes se manifestent sous forme de jaunissement suivi de flétrissement de la plante (Figure 4f), de pourriture molle sur les fruits (Figure $4 \mathrm{~g}$ ) et également de pourriture sèche du fruit (Figure 4h). 
Tableau 1 : Les différents symptômes observés sur les traitements

\begin{tabular}{|c|c|c|c|c|}
\hline $\begin{array}{c}\begin{array}{c}\text { Types de } \\
\text { traitement }\end{array} \\
\text { Symptômes } \\
\end{array}$ & Témoin & $\begin{array}{c}\text { Sciure de } \\
\text { bois chauffé }\end{array}$ & $\begin{array}{c}\text { Sciure de bois non } \\
\text { chauffée } \\
\text { additionnée de } \\
\text { pesticide }\end{array}$ & $\begin{array}{c}\text { Sciure de bois } \\
\text { chauffée } \\
\text { additionnée de } \\
\text { pesticide } \\
\end{array}$ \\
\hline $\begin{array}{l}\text { Dépérissement de } \\
\text { la tige secondaire }\end{array}$ & + & - & - & - \\
\hline $\begin{array}{l}\text { Nécroses sur la } \\
\text { feuille }\end{array}$ & + & + & - & - \\
\hline $\begin{array}{c}\text { Chancre sur le } \\
\text { collet }\end{array}$ & + & + & - & - \\
\hline $\begin{array}{c}\text { Galles sur la } \\
\text { racine }\end{array}$ & + & + & - & - \\
\hline Nécrose sur la tige & + & + & + & - \\
\hline $\begin{array}{c}\text { Jaunissement suivi } \\
\text { de flétrissement de } \\
\text { la plante }\end{array}$ & + & + & + & + \\
\hline $\begin{array}{c}\text { Pourriture molle } \\
\text { sur les fruits }\end{array}$ & + & + & + & + \\
\hline $\begin{array}{c}\text { Pourriture sèche } \\
\text { du fruit }\end{array}$ & + & + & + & + \\
\hline
\end{tabular}

+ : Présence d'un symptôme au niveau d'un traitement

- : Absence d'un symptôme au niveau d'un traitement
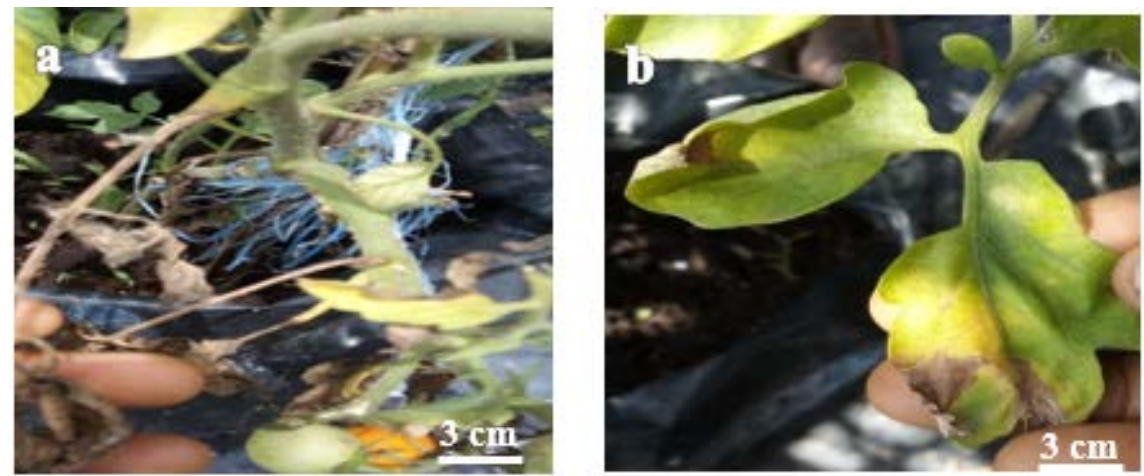

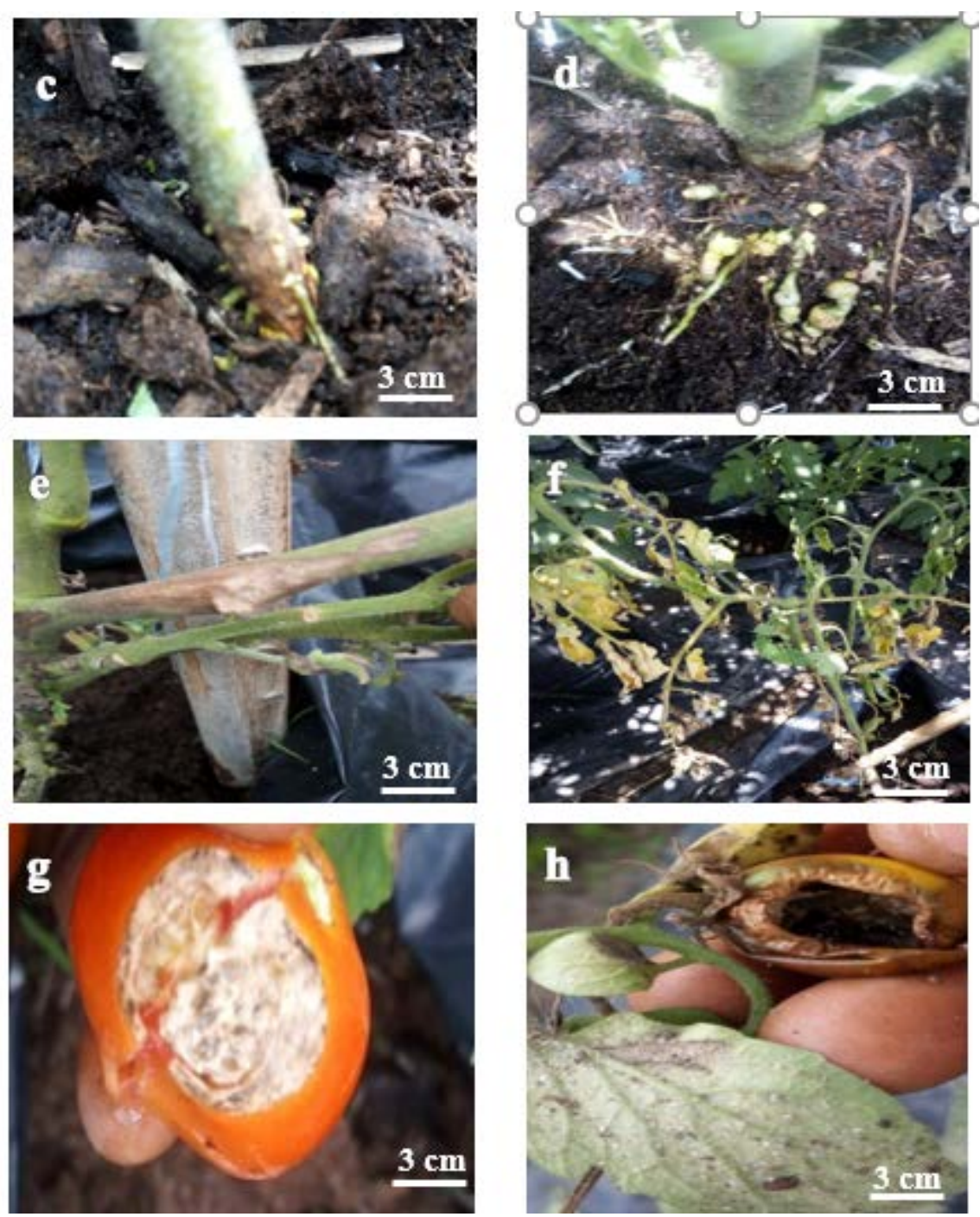

a)dépérissement de la tige secondaire, b) Nécrose sur la feuille, c) Chancre du collet, d)galle sur la racine, e) Zone morte sur la tige, f) Jaunissement du plant, g) pourriture molle du fruit, h) pourriture sèche du fruit.

Figure 5. Symptômes observés sur les plants de tomate

\section{Incidence de la maladie}

Les résultats de l'analyse statistique ont montré que l'incidence de la maladie diffère d'un traitement à l'autre $(\mathrm{P}=0,004)$. Elle augmente dans le même sens pour tous les traitements, à partir de la deuxième semaine jusqu'à la neuvième semaine après repiquage. Cependant, l'incidence est plus élevée (70 \%) dans le traitement témoin comparé aux autres traitements avec des incidences moyennes de 20,01 \% et 10,02 \% respectivement aux traitements sciure de bois chauffé et sciure de bois non chauffé additionnée de pesticide. 
Le traitement avec la sciure de bois chauffé additionnée de pesticides a montré la plus faible (5,88 \%) incidence (Figure 5).

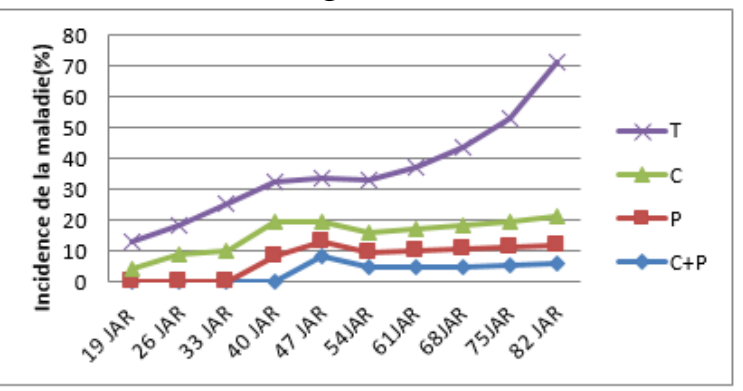

T : Traitement témoin. C : Sciure de bois chauffé. P: Sciure de bois non chauffé additionné de pesticides

$\mathbf{C}+\mathbf{P}$ : Sciure de bois chauffé additionné de pesticides. JAR : jour après repiquage

Figure 5. Effet des différents traitements sur l'incidence de la maladie

\section{Taux de mortalité des plants de tomate}

Le test de mortalité a mis en évidence un taux moyen de mortalité des plants de tomate. En effet, les taux de mortalité ont varié de 4,34 à 56,52 \% (Tableau 2). Cependant, l'analyse statistique a montré une différence significative $(P=0,003)$ entre les moyennes des différents traitements avec quatre groupes d'homogénéité. Le premier groupe comprend le traitement témoin avec la moyenne la plus élevée $(56,52 \%)$ suivie du traitement avec la sciure de bois chauffée (34,78 \%). Le troisième et quatrième groupe est constitué de la sciure de bois non chauffée additionnée de pesticides et de la sciure de bois chauffée additionnée de pesticides avec des taux de mortalités respectifs de respectivement $13,05 \%$ et $4,34 \%$.

Tableau 2 : Effet des traitements sur le taux de mortalité des plants

\begin{tabular}{|c|r|c|c|}
\hline Traitements & Taux de mortalité \pm Ecart-type & P & F \\
\hline Témoin & $56,52 \pm 4,60^{\mathbf{a}}$ & & \\
\hline $\begin{array}{c}\text { Sciure de bois non chauffée } \\
\text { additionnée de pesticide }\end{array}$ & $13,05 \pm 2,10 \mathrm{~b}^{\mathbf{b c}}$ & \multirow{2}{*}{0,003} & 5,57 \\
\hline Sciure de bois chauffée & $34,78 \pm 3,43^{\text {ab }}$ & & \\
\hline $\begin{array}{c}\text { Sciure de bois chauffée } \\
\text { additionnée de pesticide }\end{array}$ & $4,34 \pm 1,37^{\mathbf{c}}$ & & \\
\hline
\end{tabular}

P : probabilité, F: valeur de Fisher. Les moyennes affectées de la même lettre dans une colonne sont statistiquement égales au seuil de $5 \%$ selon le test de Duncan.

\section{Taux de fruits présentant des symptômes}

L'analyse statistique a montré une différence significative entre les taux moyens de fruits avariés. Trois groupes homogènes de moyennes ont été 
observés et varient de 1,96 à 72,10 \%. Le témoin a présenté le plus (72,10 \%) de fruits présentant des symptômes, suivie de la sciure de bois chauffée avec un taux de 47,61 \%. La sciure de bois non chauffé additionnée de pesticide et la sciure de bois chauffé plus ajout de pesticide ont eu respectivement un taux moyen de fruits présentant des symptômes de 4,99 et 1,96 \% (Tableau 3).

Tableau 3 : Effet des traitements sur la moyenne des fruits avariés

\begin{tabular}{|c|c|c|c|c|}
\hline Traitements & Taux de fruits \pm Ecart-type & P & & F \\
\hline Témoin & $72,10 \pm 4,8^{\mathbf{a}}$ & & & \\
\cline { 1 - 2 } $\begin{array}{c}\text { Sciure de bois non chauffée } \\
\text { additionnée de pesticide }\end{array}$ & $4,99 \pm 4,41^{\mathbf{c}}$ & \multirow{2}{*}{0,000} & \multirow{2}{*}{40,04} \\
\cline { 1 - 2 } Sciure de bois chauffée & $47,61 \pm 17,16^{\mathbf{b}}$ & & \\
\cline { 1 - 4 } $\begin{array}{c}\text { Sciure de bois chauffée } \\
\text { additionnée de pesticide }\end{array}$ & $1,96 \pm 3,40^{\mathbf{c}}$ & & \\
\hline
\end{tabular}

$\mathbf{P}$ : probabilité, F: valeur de Fisher. Les moyennes affectées de la même lettre dans une colonne sont statistiquement égales au seuil de $5 \%$ selon le test de Duncan.

\section{Discussion}

Plusieurs symptômes ont été observés sur les plants de tomate. Ce sont le dépérissement de la tige secondaire, le jaunissement et le flétrissement de la plante, les nécroses sur les feuilles et tiges, les chancres sur les collets, les galles racinaires et les pourritures molles et sèches sur les fruits. Tous les types de symptôme ont été observés sur les plants du traitement témoin. Ceux-ci pourrait être dus au fait que la sciure de bois ne soit pas chauffée, de ce fait tous les microorganismes et les insectes présents dans la sciure ont contribués à l'apparition des symptômes sur les plants. Aussi, les plants issus de tous les traitements ont présenté les symptômes. Cela pourrait être dû à la période choisie pour mener notre expérience. En effet, les températures moyennes ont varié de 28 à $29^{\circ} \mathrm{C}$ de février à mai et les précipitations de 60 à $110 \mathrm{~mm}$. Ces variations pourraient avoir un impact sur l'apparition de ces symptômes. Nos résultats confirment ceux de Torres (2010) dans ces travaux sur les systèmes alternatifs de contrôle des pathogènes telluriques en maraîchage. En effet il affirme que la prolifération de ces pathogènes seraient induite par les facteurs environnementaux tels que la pluie ou le vent qui pourraient transporter des spores de champignon sur de longues distances. L'incidence de la maladie au niveau du traitement témoin a été plus élevée comparativement à l'incidence au niveau des autres traitements. Ceci pourrait être justifié par la pression parasitaire qui serait plus forte sur les plants du traitement témoin.

Aussi, la sciure de bois chauffée additionnée de pesticides a montré la plus faible incidence. Cette faible incidence pourrait être due à l'effet du chauffage. En effet, la forte température de chauffage aurait réduit la 
population de pathogène dans la sciure. De plus, l'ajout de pesticides aurait contribué à réduire de plus l'action des pathogènes qui auraient survécu à la température de chauffage. Ces résultats corroborent ceux de Leroux (2002) qui a montré que l'utilisation des pesticides sert à détruire ou réduire les différents agents phytopathogènes. Aussi Hausbeck et al. (1996) rapportent que l'incidence de la pourriture grise et la sporulation de Botrytis cinerea sur les tiges et les feuilles du géranium dans des serres commerciales a été significativement réduite par rapport au témoin non chauffé.

Tout comme la variation de l'incidence, le taux de mortalité des plants de tomate et le taux de fruits avariés ont été plus élevés avec le traitement témoin que celui avec la sciure de bois chauffée additionnée de pesticides. Ce constat pourrait également être dû à l'action de la température de chauffage de la sciure de bois couplée avec l'apport des pesticides. En effet, les pesticides auraient contribué à réduire l'action des pathogènes ayant survécu à la température.

En effet, selon Boa (2016), les fruits avariés pourraient résulter d'une part, des indicateurs de sensibilité des fruits aux maladies et d'autre part à l'attaque des insectes. Ces résultats coïncident avec ceux de Zuang et al. (1996) et Miller et al. (1997) qui ont révélé que le compost peut améliorer l'aptitude des plantes à résister aux maladies causées par les agents pathogènes foliaires en induisant une résistance systémique.

\section{Conclusion}

L'efficacité des traitements de la sciure de bois ont été observé sur la variété F1 COBRA 26. Le traitement avec la sciure de bois chauffée additionnée de pesticides a montré une faible incidence, un faible taux de mortalité et un faible taux de fruits avariés comparativement aux traitements témoin, sciure de bois chauffé et sciure de bois non chauffée additionnée de pesticides. Ce résultat nous permet de suggérer que l'utilisation de la sciure de bois chauffé plus ajout des pesticides a un effet important sur le contrôle de la pathologie comparé aux autres traitements. Ainsi l'utilisation de la sciure de bois chauffé avec des pesticides a été efficace pour la lutte contre les pathogènes de la tomate.

\section{References:}

1. Adjiri O.A., Kone B., Aka N., Djabakate I. \& Dibi B. (2019). Caractérisation physico-chimique et source de la minéralisation des eaux souterraines des départements de Daloa et Zoukougbeu, Côte d'Ivoire. Revue Internationale des sciences biologiques et chimiques, 13(4): 2392-2393

2. Anonyme. (2005). Appui à la mise en œuvre du NEP AD-PDDAA Profil de projet d'investissement bancable en Côte d'Ivoire, 
production de la banane plantain et aux productions maraîchères en zone de forêt. Rapport de l'organisation des nations unies pour l'alimentation et l'agriculture (FAO), $7 \mathrm{p}$.

3. Bennett A.J. \& Whipps J.M. (2003). Survival of the biocontrol agents Coniothyrium minitans and Bacillus subtilis MBI 600 introduced into pasteurised, sterilised and non-sterile soils. Soil Biology \& Biochemistry, 15(3): 1565-1573.

4. Bernier P. (2015). Vers la construction d'un discours critique de l'agriculture urbaine commerciale en serres sur les toits. Mémoire de la maitrise en sociologie, UFR de Sociologie, Université du Québec (Montréal, Canada), 183 p.

5. Biekre A.H.T. (2013). Evaluation de quelques paramètres agronomiques de variétés de tomate [Solanum lycopersicum L. (Solanaceae)] introduites en Côte d'Ivoire. Mémoire de Master, Option : Biologie et Protection des Végétaux, UFR Science de la Nature, Université Nangui Abrogoua, (Abobo, Côte d'Ivoire), 59 p.

6. Boa B.B.Y. (2016). Effet de trois substrats à base de sous-produits locaux sur deux variétés de tomate en culture hydroponique au centre de la Côte d'Ivoire. Mémoire de master en Environnement, Laboratoire : Géosciences et Environnement, Université Nangui Abrogoua (Abobo, Côte d'Ivoire), 48 p.

7. Djaha K.F. (2015). Contribution de nouveaux modes de production a une agriculture durable : cas de l'agriculture hors sol en Côte d'Ivoire. Mémoire de licence Professionnelle en Science et Gestion de l'Environnement, Option: Ecologie et Environnement en milieux rural, UFR Science et Gestion de l'Environnement, Université Nangui Abrogoua (Abobo, Côte d'Ivoire), 41p.

8. Hausbeck M.K., Pennypacker S.P. \& Stevenson R.E. (1996). The effect of plastic mulch and forced heated air on Botrytis cinerea on geranium stock plants in a research greenhouse. Plant Disease, 80: 170-173.

9. INRA. (2010). Importance économique de la tomate. Rapport d'étude de l'Institut National Agronomique, 14 p.

10. Leroux P., Delorme R., Gaillardon P. (2002). Évolutions des produits phytosanitaires à usage agricoles, les fongicides. Edition Gestion Location Intervention, Paris (France), pp 8-15.

11. Miller S.A., Sabin F., Krause M., AI-Dahmani J., Stone A. \& Hoitink H. (1997). Control of bacterial leaf spot of radish in compost-amended planting mixes. Journal of Phytopathology, 3: 66-87. 
12. Naika S., Jeude J.V.L., Goffau M., Hilmi M. \& Dam B.V. (2005). La culture de la tomate production, transformation et commercialisation. Ed. Prota, France, 105 p.

13. Nechadi S., Benddine F., Moumen A. \& Kheddam.M. (2002). Etat des maladies virales de la tomate et stratégie de lutte en Algérie, $21 \mathrm{p}$.

14. N'zi J.C., Kouame C., Assanvo S.P.N., Fondio L., Djidji A.H. \& Sangare A. (2010). Evolution des populations de Bemisia tabaci Genn. Selon les variétés de tomates (Solanum lycopersicum L.) au centre de la Côte d'Ivoire, Sciences et Nature, 7(1) : 31-40.

15. Sangaré A., Koffi E., Akamou F. \& Fall C.A. (2009). L'état des ressources phylogénétiques pour l'alimentation et l'agriculture, Rapport national, Côte d'Ivoire, 65 p.

16. Sedki M., Mimouni A. (1995). Effets de substrats locaux sur tomate en culture hors sol, INRA, Al Awamia, Souss-sahara (Maroc), $15 \mathrm{p}$.

17. Soro S. (2009). Caractérisation agronomique et culture sous voile de variétés améliorées de tomate (Lycopersicum esculentum Mill) en Côte d'Ivoire. Thèse de Doctorat d'Etat, UFR Science Naturel Université d'Abobo-Adjamé, (Abidjan, Côte d'Ivoire), 156 p.

18. Torres M. (2010). Les systèmes alternatifs de contrôle des pathogènes telluriques en maraîchage, Analyse des modalités techniques et évaluation des risques d'infestations en exploration agricole. Mémoire de fin d'études, filière Agriculture, Ecole d’ingénieur ISRA-Lyon, France, 116 p.

19. Vakalounakis D.J. \& Fragkiadakis G.A. (1999). Genetic diversity of Fusarium oxysporum isolates from cucumber, differentiation by pathogenicity, vegetative compatibility and RAPD fingerprinting. Phytopathology, 89(2): 161- 168.

20. Zuang H. \& Musard M. (1986). Cultures légumières sur substrats : Installation et conduite. Paris (France), 276 p. 